\title{
On generalized solutions for discontinuous fuzzy differential equations and strong fuzzy Henstock integrals
}

\author{
Ya-Bin Shao ${ }^{a}$, Zeng-Tai Gong ${ }^{b, *}$, Zi-Zhong Chen ${ }^{c}$ \\ a School of Science, Chongqing University of Posts and Telecommunications, Nanan, 400065, Chongqing, P. R. China. \\ ${ }^{b}$ College of Mathematics and Statistics, Northwest Normal University, Lanzhou 730070, Gansu, P. R. China. \\ ${ }^{c}$ College of Computer Science and Technology, Chongqing University of Posts and Telecommunications, Nanan, 400065, Chongqing, \\ P. R. China.
}

Communicated by $\mathrm{X}$. Liu

\begin{abstract}
In this paper, under the notion of strong uniformly $A C^{\nabla}$ of fuzzy-number-valued functions, we prove a generalized controlled convergence theorem of strong fuzzy Henstock integral. As the applications of this convergence theorem, we provide sufficient conditions which guarantee the existence of generalized solutions to initial value problems for the fuzzy differential equations by using properties of strong fuzzy Henstock integrals under strong GH-differentiability. In comparison with some previous works, we consider equations whose right-hand side functions are not integrable in the sense of Kaleva on certain intervals and their solutions are not absolute continuous functions. (C)2017 All rights reserved.
\end{abstract}

Keywords: Fuzzy number, strong fuzzy Henstock integral, generalized controlled convergence theorem, fuzzy differential equations, generalized solution.

2010 MSC: 03E72, 26A39, 46G05.

\section{Introduction}

It is well-known that the Henstock integral includes the Riemann, improper Riemann, Lebesgue and Newton integrals. Though such an integral was defined by Denjoy in 1912 and also by Perron in 1914, it was difficult to handle using their definitions. But with the Riemann-type definition introduced more recently by Henstock [16] in 1963 and also independently by Kurzweil [20], the definition is now simple and furthermore the proof involving the integral also turns out to be easy. For more detailed results about the Henstock integral, we refer to [23]. The integrals of fuzzy-number-valued functions, as a natural generalization of set-valued functions, have been discussed by Puri and Ralescu [25], Kaleva [17], and other authors [28, 30,32]. Recently, Wu and Gong [13, 15, 31] have combined the fuzzy set theory [34] and nonabsolute integration theory [23], and discussed the fuzzy Henstock integrals of fuzzy-numbervalued functions which extended Kaleva [17] integration. Furthermore, in order to complete the theory

\footnotetext{
${ }^{*}$ Corresponding author Chen)

Email addresses: yb-shao@163.com (Ya-Bin Shao), zt-gong@163.com (Zeng-Tai Gong), chenzz@cqupt.edu.cn (Zi-Zhong 
of fuzzy calculus and to meet the solving need of transferring a fuzzy differential equation into a fuzzy integral equation, we $[13,15]$ has defined the strong fuzzy Henstock integrals and discussed some of their properties and the controlled convergence theorem.

In 1972, Chang and Zadeh [6] first introduced the concept of fuzzy derivative, followed up ten years later by Dubois and Prade [10], who used the extension principle in their approach. In the mean time, Puri and Ralescu [25] used the notion of H-differentiability to extend the differential of set-valued functions to that of fuzzy functions. This led Seikkala [27] to introduce the notion of fuzzy derivative as an extension of the Hukuhara derivative and the fuzzy integral, which was the same as that proposed by Dubois and Prade [11, 12]. Naturally, the investigation of fuzzy differential and integral equations, existence and uniqueness theorems for the solutions of fuzzy initial value problems, drew upon the interest of many researchers of the fuzzy domain (see [1, 3, 4, 7, 8, 14, 18, 19, 22, 24, 29]). In 2002, Xue and Fu [33] established solutions to fuzzy differential equations with right-hand side functions satisfying Caratheoedory conditions on a class of Lipschitz fuzzy sets. However, there are discontinuous systems in which the right-hand side functions are not integrable in the sense of Kaleva [17] on certain intervals and their solutions are not absolute continuous functions.

The paper is organized as follows. In Section 2, we collect some basic concepts and preliminary results of interest and then, in Section 3, we study the generalized convergence theorem of strong fuzzy Henstock integral under the notion of strong uniformly $A C^{\nabla}$ of fuzzy-number-valued functions. In Section 4 , as the applications of the generalized convergence theorem, we provide the existence of generalized solutions to initial value problems for the fuzzy differential equations. Finally, in Section 5, we give some concluding remarks.

\section{Preliminaries}

The space of fuzzy numbers is defined as follows: $\mathbb{R}_{F}=\left\{u: R^{n} \rightarrow[0,1] \mid u\right.$ satisfies (1)-(4) below $\}$ is a fuzzy number space, where

(1) $u$ is normal, i.e., there exists an $x_{0} \in R^{n}$ such that $u\left(x_{0}\right)=1$;

(2) $u$ is fuzzy convex, i.e., $u(\lambda x+(1-\lambda) y) \geqslant \min \{u(x), u(y)\}$ for any $x, y \in R^{n}$ and $0 \leqslant \lambda \leqslant 1$;

(3) $u$ is upper semi-continuous;

(4) $[u]^{0}=\operatorname{cl}\left\{x \in R^{\mathfrak{n}} \mid \mathfrak{u}(x)>0\right\}$ is compact.

For $0<\alpha \leqslant 1$, denote $[u]^{\alpha}=\left\{x \in R^{\mathfrak{n}} \mid \mathfrak{u}(x) \geqslant \alpha\right\}$. Then from above (1)-(4), it follows that the $\alpha$-level set $[u]^{\alpha} \in P_{k}\left(R^{n}\right)$ for all $0 \leqslant \alpha<1$.

The metric in $\mathbb{R}_{\mathrm{F}}$ is defined by $\mathrm{D}: \mathbb{R}_{\mathrm{F}} \times \mathbb{R}_{\mathrm{F}} \rightarrow[0, \infty)$

$$
\mathrm{D}(\mathrm{u}, v)=\sup \left\{\mathrm{d}_{\mathrm{H}}\left([\mathrm{u}]^{\alpha},[v]^{\alpha}\right): \alpha \in[0,1]\right\},
$$

where $d_{H}$ is the Hausdorff metric defined in $P_{k}\left(R^{n}\right)$, where $P_{k}\left(R^{n}\right)$ denotes the compact convex subsets of $R^{n}$. Then it is easy to see that $D$ is a metric in $\mathbb{R}_{F}$. Using the results [9], we know that

(1) $\left(\mathbb{R}_{\mathrm{F}}, \mathrm{D}\right)$ is a complete metric space;

(2) $\mathrm{D}(u+w, v+w)=\mathrm{D}(u, v)$ for all $u, v, w \in \mathbb{R}_{\mathrm{F}}$;

(3) $\mathrm{D}(\lambda u, \lambda v)=|\lambda| \mathrm{D}(u, v)$ for all $u, v, w \in \mathbb{R}_{\mathrm{F}}$ and $\lambda \in R$;

(4) $\mathrm{D}(u+v, \tilde{0}) \leqslant \mathrm{D}(u, \tilde{0})+\mathrm{D}(u, \tilde{0})$.

According to Zadeh's extension principle, we have addition and scalar multiplication in fuzzy number space $\mathbb{R}_{\mathrm{F}}$ as follows [10]:

$$
[u+v]^{\alpha}=[u]^{\alpha}+[v]^{\alpha}, \quad[k u]^{\alpha}=k[u]^{\alpha},
$$

where $u, v \in \mathbb{R}_{\mathrm{F}}$ and $0 \leqslant \alpha \leqslant 1$. 
We will consider the partial order $\preceq$ defined as follows: if $u, v \in \mathbb{R}_{\mathrm{F}}$ with $[u]^{\alpha}=\left[u_{l}^{\alpha}, u_{r}^{\alpha}\right]$ and $[v]^{\alpha}=$ $\left[v_{l}^{\alpha}, v_{r}^{\alpha}\right]$, then

$$
u \preceq v \Leftrightarrow u_{l}^{\alpha} \leqslant v_{l}^{\alpha} \quad \text { and } \quad u_{r}^{\alpha} \leqslant v_{r}^{\alpha}, \quad \alpha \in[0,1] .
$$

A fuzzy-number-valued function $\tilde{f}:[a, b] \rightarrow \mathbb{R}_{\mathrm{F}}$ is said to satisfy the condition $(\mathrm{H})$ on $[a, b]$, if for any $x_{1}<x_{2}$ belonging to $[a, b]$ there exists $\tilde{u} \in \mathbb{R}_{F}$ such that $\tilde{f}\left(x_{2}\right)=\tilde{f}\left(x_{1}\right)+\tilde{u}$. We call $\tilde{u}$ is the H-difference of $\tilde{f}\left(x_{2}\right)$ and $\tilde{f}\left(x_{1}\right)$, denoted by $\tilde{f}\left(x_{2}\right) \ominus_{H} \tilde{f}\left(x_{1}\right)$ (see [17]).

It is well-known that the H-derivative for fuzzy-number-functions was initially introduced by Puri and Ralescu [26] and it is based on the condition $(\mathrm{H})$ of sets. We note that this definition is fairly strong, because the family of fuzzy-number-valued functions $\mathrm{H}$-differentiable is very restrictive. For example, the fuzzy-number-valued function $\tilde{f}:[a, b] \rightarrow \mathbb{R}_{F}$ defined by $\tilde{f}(x)=C \cdot g(x)$, where $C$ is a fuzzy number, "." is the scalar multiplication (in the fuzzy context) and $g:[a, b] \rightarrow R^{+}$, with $g^{\prime}\left(t_{0}\right)<0$, is not H-differentiable in $t_{0}$ (see $\left.[2,5]\right)$. To avoid the above difficulty, in this paper we consider a more general definition of a derivative for fuzzy-number-valued functions enlarging the class of differentiable fuzzy-number-valued functions, which has been introduced in [2].

Definition $2.1([2])$. Let $\tilde{f}:(a, b) \rightarrow \mathbb{R}_{\mathrm{F}}$ and $x_{0} \in(a, b)$. We say that $\tilde{f}$ is $(\mathfrak{i})$-differentiable at $x_{0}$, if there exists an element $\tilde{f}^{\prime}\left(t_{0}\right) \in \mathbb{R}_{F}$, such that for all $h>0$ sufficiently small, there exists $\tilde{f}\left(x_{0}+h\right) \ominus_{H}$ $\tilde{f}\left(x_{0}\right), \tilde{f}\left(x_{0}\right) \ominus_{H} \tilde{f}\left(x_{0}-h\right)$ and the limits (in the metric D)

$$
\lim _{h \rightarrow 0} \frac{\tilde{f}\left(x_{0}+h\right) \ominus_{H} \tilde{f}\left(x_{0}\right)}{h}=\lim _{h \rightarrow 0} \frac{\tilde{f}\left(x_{0}\right) \ominus_{H} \tilde{f}\left(x_{0}-h\right)}{h}=\tilde{f}^{\prime}\left(x_{0}\right) .
$$

$\tilde{f}$ is $(i i)$-differentiable at $x_{0}$, for all $h<0$ sufficiently small, there exists $\tilde{f}\left(x_{0}+h\right) \ominus_{H} \tilde{f}\left(x_{0}\right), \tilde{f}\left(x_{0}\right) \ominus_{H} \tilde{f}\left(x_{0}-h\right)$ and the limits (in the metric D)

$$
\lim _{h \rightarrow 0} \frac{\tilde{f}\left(x_{0}+h\right) \ominus_{H} \tilde{f}\left(x_{0}\right)}{h}=\lim _{h \rightarrow 0} \frac{\tilde{f}\left(x_{0}\right) \ominus_{H} \tilde{f}\left(x_{0}-h\right)}{h}=\tilde{f}^{\prime}\left(x_{0}\right) .
$$

\section{Strong fuzzy Henstock integral in fuzzy number space}

In this section we define the strong fuzzy Henstock integral in fuzzy number space and give a generalized convergence theorem for this integral.

Definition 3.1 ([23]). Let $\delta(\xi)$ be a positive real function on a closed set $[a, b]$. A division $P=\left\{\left(\xi_{i},\left[x_{i-1}, x_{i}\right]\right)\right\}$ is said to be $\delta$-fine, if the following conditions are satisfied:

(1) $a=x_{1}<x_{2}<\cdots<x_{n}=b$;

(2) $\xi_{i} \in\left[x_{i-1}, x_{i}\right] \subset\left(\xi_{i}-\delta\left(\xi_{i}\right), \xi_{i}+\delta\left(\xi_{i}\right)\right)$.

Definition 3.2 ([15]). A fuzzy-number-valued function $\tilde{f}$ will be termed additive on $[a, b]$ if for any division $T: a \leqslant x_{1} \leqslant x_{2} \leqslant \cdots \leqslant x_{n} \leqslant b$, we have $\tilde{f}\left(\left[x_{i}, x_{j}\right]\right)(1 \leqslant i<j \leqslant n)$ exists and $\tilde{f}\left(\left[x_{i}, x_{j}\right]\right)=$ $\sum_{k=i}^{j-1} \tilde{f}\left(\left[x_{k}, x_{k+1}\right]\right)$ or $\tilde{f}\left(\left[x_{j}, x_{i}\right]\right)(1 \leqslant i<j \leqslant n)$ exists and $(-1) \cdot \tilde{f}\left(\left[x_{j}, x_{i}\right]\right)=(-1) \cdot \sum_{k=i}^{j-1} \tilde{f}\left(\left[x_{k+1}, x_{k}\right]\right)$. For convenience, denote $\tilde{f}([s, t])$ by $\tilde{f}(t) \ominus_{H} \tilde{f}(s)$.

Definition 3.3 ([13, 15]). A fuzzy-number-valued function $\tilde{f}$ is said to be strong Henstock integrable on $[a, b]$ if there exists an additive fuzzy-number-valued function $\tilde{F}$ on $[a, b]$ such that for every $\varepsilon>0$ there is a function $\delta(\xi)>0$ and for any $\delta$-fine division $P=\{([u, v], \xi)\}$ of $[a, b]$, we have

$$
\sum_{i \in K_{n}} \mathrm{D}\left(\tilde{\mathrm{f}}\left(\xi_{i}\right)\left(v_{i}-u_{i}\right), \tilde{\mathrm{F}}\left(\left[u_{i}, v_{i}\right]\right)\right)+\sum_{j \in I_{n}} \mathrm{D}\left(\tilde{f}\left(\xi_{j}\right)\left(v_{j}-u_{j}\right),(-1) \cdot \tilde{F}\left(\left[u_{j}, v_{j-1}\right]\right)\right)<\varepsilon,
$$

where $K_{n}=\{i \in\{1,2, \cdots, n\}\}$ such that $\tilde{F}\left(\left[x_{i-1}, x_{i}\right]\right)$ is a fuzzy number and $I_{n}=\{j \in\{1,2, \cdot \cdot, n\}\}$ such that $\tilde{F}\left(\left[x_{j}, x_{j-1}\right]\right)$ is a fuzzy number. We write $\tilde{f} \in \operatorname{SFH}[a, b]$. 
Definition 3.4. A sequence of continuous fuzzy-number-valued functions $\tilde{F}_{n}:[a, b] \rightarrow \mathbb{R}_{F}$ is said to be uniformly negligible variation on a set $Z \subset[a, b]$ if for every $\varepsilon>0$ there exists $\delta(\xi)>0, \xi \in Z$ such that if $\left\{\left(\xi_{i},\left[u_{i}, v_{i}\right]\right)\right\}$ is a $\delta$-fine division with $\xi_{i} \in Z$ then

$$
\sum \mathrm{D}\left(\tilde{\mathrm{F}}_{\mathrm{n}}\left(\left[\mathrm{u}_{i}, v_{i}\right]\right), \tilde{0}\right)<\varepsilon
$$

for all $n \in \mathbb{N}$.

Theorem $3.5([15])$. Let $\tilde{\mathrm{f}}:[\mathrm{a}, \mathrm{b}] \rightarrow \mathbb{R}_{\mathrm{F}}$. If $\tilde{\mathrm{f}}=0$ a.e. on $[\mathrm{a}, \mathrm{b}]$, then $\tilde{\mathrm{f}}$ is SFH integrable on $[\mathrm{a}, \mathrm{b}]$ and $\int_{a}^{b} \tilde{f}(t) d t=0$.

Definition 3.6 ([15]). A sequence of fuzzy-number-valued functions $\left\{\tilde{f_{n}}\right\}$ is said to be strong fuzzy Henstock equi-integrable on $[a, b]$, if $\tilde{f_{n}}, n=1,2, \cdots$, is strong fuzzy Henstock integrable on $[a, b]$ and for any $\varepsilon>0$ there is a function $\delta(\xi)>0$ and for any $\delta$-fine division $\left\{\left(\xi_{i},\left[u_{i}, v_{i}\right]\right)\right\}$ such that

$$
\sum D\left(\tilde{f}\left(\xi_{i}\right)\left(v_{i}-u_{i}\right), \int_{a}^{b} \tilde{f}\right)<\varepsilon
$$

We have the following simple result.

Theorem 3.7. Let $\left\{\tilde{f}_{n}\right\}$ be a sequence of strong fuzzy Hentock integrable functions such that $\lim _{n \rightarrow \infty} \tilde{f}_{n}(x)=\tilde{f}(x)$ for all $\mathrm{x} \in[\mathrm{a}, \mathrm{b}]$ and let $\tilde{\mathrm{F}}_{\mathrm{n}}(\mathrm{x})=\int_{\mathrm{a}}^{\mathrm{x}} \tilde{\mathrm{f}}_{\mathrm{n}}$. If there is a set $\mathrm{Z} \subset[\mathrm{a}, \mathrm{b}], \mu(\mathrm{Z})=0$ such that

(i) the sequence $\tilde{F}_{n}$ is uniformly differentiable to $\tilde{f}_{n}$ on $[a, b] \backslash Z$;

(ii) the sequence $\tilde{\mathrm{F}}_{\mathrm{n}}$ is uniformly negligible variation on $\mathrm{Z}$,

then the function $\tilde{\mathrm{f}}(\mathrm{x})$ is strong fuzzy Henstock integrable and

$$
\lim _{n \rightarrow \infty} \int_{a}^{b} \tilde{f}_{n}(x) d x=\int_{a}^{b} \tilde{f}(x) d x .
$$

Proof. Define $\tilde{f}_{n}^{Z}(x)=\tilde{f}_{n}(x)$ for $x \in[a, b] \backslash Z$ and $\tilde{f}_{n}^{Z}(x)=\tilde{0}$ for $x \in Z$. Then $\lim _{n \rightarrow \infty} \tilde{f}_{n}^{Z}(x)=\tilde{f}^{Z}(x)$ for every $x \in[a, b]$ where $\tilde{f}^{Z}(x)=\tilde{f}(x)$ for $x \in[a, b] \backslash Z$ and $\tilde{f}^{Z}(x)=\tilde{0}$ for $x \in Z$. Since $\tilde{f}_{n}^{Z}(x)=\tilde{f}_{\mathfrak{n}}(x)$ a.e. on $[a, b]$ we have by Theorem 3.5 the strong fuzzy Henstock integrability of $\tilde{f}_{n}^{Z}$ and $\tilde{F}_{n}(x)=\int_{a}^{x} \tilde{f}_{n}=\int_{a}^{x} \tilde{f}_{n}^{Z}$ for $x \in[a, b]$.

Now, we consider

$$
\begin{aligned}
\sum \mathrm{D}\left(\tilde{f}_{n}^{Z}\left(\xi_{i}\right)\left(v_{i}-u_{i}\right), \tilde{F}_{n}\left(\left[u_{i}, v_{i}\right]\right)\right) \leqslant & \sum_{\xi_{i} \in[a, b] \backslash Z} D\left(\tilde{f}_{n}^{Z}\left(\xi_{i}\right)\left(v_{i}-u_{i}\right), \tilde{F}_{n}\left(\left[u_{i}, v_{i}\right]\right)\right) \\
& +\sum_{\xi_{i} \in Z} D\left(\tilde{f}_{n}^{Z}\left(\xi_{i}\right)\left(v_{i}-u_{i}\right), \tilde{F}_{n}\left(\left[u_{i}, v_{i}\right]\right)\right) .
\end{aligned}
$$

For $\xi_{i} \in[a, b] \backslash Z$, by condition (i) we have

$$
\begin{aligned}
\mathrm{D}\left(\tilde{\mathrm{f}}_{\mathfrak{n}}^{\mathrm{Z}}\left(\xi_{i}\right)\left(v_{i}-u_{i}\right), \tilde{\mathrm{F}}_{\mathfrak{n}}\left(\left[u_{i}, v_{i}\right]\right)\right) \leqslant & \mathrm{D}\left(\tilde{\mathrm{f}}_{\mathfrak{n}}^{\mathrm{Z}}\left(\xi_{i}\right)\left(v_{i}-\xi_{i}\right), \tilde{\mathrm{F}}_{\mathfrak{n}}\left(\left[u_{i}, v_{i}\right]\right)\right) \\
& +\mathrm{D}\left(\tilde{\mathrm{f}}_{\mathfrak{n}}^{\mathrm{Z}}\left(\xi_{i}\right)\left(\xi_{i}-u_{i}\right), \tilde{\mathrm{F}}_{\mathfrak{n}}\left(\left[u_{i}, v_{i}\right]\right)\right) \\
& <2 \varepsilon\left(v_{i}-u_{i}\right)
\end{aligned}
$$

while for $\xi_{i} \in Z$, condition (ii) and $\tilde{f}_{\mathfrak{n}}^{Z}\left(\xi_{i}\right)=\tilde{0}$ yields

$$
\sum_{\xi_{i} \in Z} D\left(\tilde{f}_{n}^{Z}\left(\xi_{i}\right)\left(v_{i}-u_{i}\right), \tilde{F}_{n}\left(\left[u_{i}, v_{i}\right]\right)\right)=\sum D\left(\tilde{F}_{n}\left(\left[u_{i}, v_{i}\right]\right), \tilde{0}\right)<\varepsilon
$$


Hence

for any $n \in \mathbb{N}$.

$$
\sum D\left(\tilde{f}_{n}^{Z}\left(\xi_{i}\right)\left(v_{i}-u_{i}\right), \tilde{F}_{n}\left(\left[u_{i}, v_{i}\right]\right)\right) \leqslant 2 \varepsilon \sum\left(v_{i}-u_{i}\right)+\varepsilon=2 \varepsilon(b-a)+\varepsilon
$$

By Definition 3.6 this means that the sequence $\left\{\tilde{f}_{n}^{Z}\right\}$ is equi-integrable and [15, Theorem 5.1] gives the strong fuzzy Henstock integrability of the function $\tilde{f}^{\mathrm{Z}}$ and

$$
\lim _{n \rightarrow \infty} \int_{a}^{b} \tilde{f}_{n}^{Z}(x) d x=\int_{a}^{b} \tilde{f}^{Z}(x) d x
$$

Since $\tilde{f}_{n}^{Z}, \tilde{f}^{Z}$ differ from $\tilde{f}_{n}, \tilde{f}$ on the set $Z$ with $\mu(Z)=0$ only, Theorem 3.5 gives the result.

Now we present a convergence theorem for a sequence of pointwise convergent strong fuzzy Henstock integrable functions using conditions on the sequence of their primitives.

Definition 3.8 ([21]). If $\left\{\left(\xi_{i},\left[u_{i}, v_{i}\right]\right)\right\}$ and $\left\{\left(\zeta_{j},\left[s_{j}, t_{j}\right]\right)\right\}$ are two $\delta$-fine division in $[a, b]$ and $\mu\left(\cup_{i}\left[u_{i}, v_{i}\right] \triangle \cup_{j}\right.$ $\left.\left[s_{j}, t_{j}\right]\right) \leqslant \eta$, then $\left\{\left(\xi_{i},\left[u_{i}, v_{i}\right]\right)\right\}$ and $\left\{\left(\zeta_{j},\left[s_{j}, t_{j}\right]\right)\right\}$ are said to be $\eta$-close.

Note that the symmetric difference of two sets is denoted by $\triangle$.

Definition 3.9. A fuzzy-number-valued function $\tilde{F}$ defined on $X \subset[a, b]$ is said to be strong $A C^{\nabla}(X)$ if for every $\varepsilon>0$ there exists $\eta>0$ such that

$$
\left|\sum_{i} \mathrm{D}\left(\tilde{\mathrm{F}}\left(\left[u_{i}, v_{i}\right]\right), \tilde{0}\right)-\sum_{j} \mathrm{D}\left(\tilde{\mathrm{F}}\left(\left[s_{j}, \mathbf{t}_{j}\right]\right), \tilde{0}\right)\right|<\varepsilon,
$$

for any two $\eta$-close $\delta$-fine division $\left\{\left(\xi_{i},\left[u_{i}, v_{i}\right]\right)\right\}$ and $\left\{\left(\zeta_{j},\left[s_{j}, t_{j}\right]\right)\right\}$.

Definition 3.10. A fuzzy-number-valued function $\tilde{F}_{n}$ is said to be uniformly strong $A C^{\nabla}$ on $X$ if for every $\varepsilon>0$ there exists $\eta>0$ such that

$$
\left|\sum_{i} \mathrm{D}\left(\tilde{\mathrm{F}}_{\mathfrak{n}}\left(\left[u_{i}, v_{i}\right]\right), \tilde{0}\right)-\sum_{j} \mathrm{D}\left(\tilde{\mathrm{F}}_{\mathfrak{n}}\left(\left[\mathrm{s}_{j}, \mathrm{t}_{j}\right]\right), \tilde{0}\right)\right|<\varepsilon,
$$

for any two $\eta$-close $\delta$-fine division $\left\{\left(\xi_{i},\left[u_{i}, v_{i}\right]\right)\right\}$ and $\left\{\left(\zeta_{j},\left[s_{j}, t_{j}\right]\right)\right\}$ and all $n \in \mathbb{N}$.

Theorem 3.11. If there exists a fuzzy-number-valued function $\tilde{\mathrm{F}}$ which is continuous and strong $\mathrm{AC}^{\nabla}$ on $[\mathrm{a}, \mathrm{b}]$ such that $\tilde{\mathrm{F}}^{\prime}(\mathrm{x})=\tilde{\mathrm{f}}(\mathrm{x})$ a.e. in $[\mathrm{a}, \mathrm{b}]$, then $\tilde{\mathrm{f}}$ is SFH integrable on $[\mathrm{a}, \mathrm{b}]$ with primitive $\tilde{\mathrm{F}}$.

Let us start with a few lemmas.

Lemma 3.12 ([21]). Assume that $p:[a, b] \rightarrow(0,+\infty), Z \in[a, b], \mu(Z)=0$ and $\varepsilon>0$. Then there is $\delta(\xi)>0$ and for any $\delta$-fine division such that

$$
\sum_{i} p\left(\xi_{i}\right)\left(v_{i}-u_{i}\right)<\varepsilon
$$

with $\xi_{i} \in Z$.

Lemma 3.13. Let $\tilde{f}_{n}:[a, b] \rightarrow \mathbb{R}_{F}$ be such that $\lim _{n \rightarrow \infty} \tilde{f}_{n}(x)=\tilde{f}(x)$ for every $x \in[a, b], Z \subset[a, b]$. Then for every $\varepsilon>0$ there exists $\delta(\xi)>0$ and for any $\delta$-fine division such that

$$
\sum_{i} \mathrm{D}\left(\tilde{f}_{n}\left(\xi_{i}\right)\left(v_{i}-u_{i}\right), \tilde{0}\right)<\varepsilon
$$

with $\xi_{i} \in Z$.

Proof. Define $p(t)=\sup _{n \in \mathbb{N}} D\left(\tilde{f}_{n}, \tilde{0}\right)$. Since the sequence $\tilde{f}_{m}$ converges pointwise in $\mathbb{R}_{F}$ we have $p:[a, b] \rightarrow$ $(0,+\infty)$. Using Lemma 3.12 we have

$$
\sum_{i} D\left(\tilde{f}_{n}\left(\xi_{i}\right)\left(v_{i}-u_{i}\right), \tilde{0}\right) \leqslant \sum_{i} p\left(\xi_{i}\right)\left(v_{i}-u_{i}\right)<\varepsilon
$$

which completes the proof. 
Lemma 3.14. Let $X_{1} \subset[a, b]$ be closed, $X_{2} \subset[a, b]$ and let the sequence of additive functions $\tilde{F}_{n}$ be strong uniformly $A C^{\nabla}\left(X_{m}\right)$ for $m=1,2$. Then $\tilde{\mathrm{F}}_{n}$ is uniformly strong $A C^{\nabla}\left(X_{1} \cup X_{2}\right)$.

Lemma 3.15. Let $\tilde{\mathrm{F}}_{\mathrm{n}}$ be additive fuzzy-number-valued functions. Assume that a sequence $X_{\mathrm{m}} \subset[\mathrm{a}, \mathrm{b}], \mathrm{m} \in \mathbb{N}$ of measurable sets such that $\cup_{m} X_{m}=[a, b]$ and sequence $\tilde{F}_{n}$ is uniformly strong $A C^{\nabla}\left(X_{m}\right)$. Let $Z \subset[a, b], \mu(Z)=$ 0 . Then for every $\varepsilon>0$ there exists $\delta(\xi)>0$ such that

$$
\sum_{i} \mathrm{D}\left(\tilde{\mathrm{F}}_{\mathrm{n}}\left(\left[\mathrm{u}_{\mathrm{i}}, v_{\mathrm{i}}\right]\right), \tilde{0}\right)<\varepsilon
$$

if $\left\{\left(\xi_{i},\left[u_{i}, v_{i}\right]\right)\right\}$ is a $\delta$-fine division with $\xi_{i} \in Z$.

Proof. Let $\varepsilon>0$ be given and assume without loss of generality that the set $X_{m}$ are pairwise disjoint. By Definition 3.9, there exist $\delta_{m}(\xi)>0$ and $\eta_{m}>0$ such that

$$
\sum_{i} \mathrm{D}\left(\tilde{\mathrm{F}}_{n}\left(\left[u_{i}, v_{i}\right]\right), \tilde{0}\right)<\frac{\varepsilon}{2^{m}}
$$

for any $\delta_{m}$-fine division with $\xi_{i} \in X_{m}$ and $\mu\left(\cup_{i}\left[u_{i}, v_{i}\right]\right)<\eta_{m}$. Since $\mu\left(X_{m} \cap Z\right)=0$, there exist open sets $\mathrm{H}_{\mathrm{m}} \subset \mathbb{R}$ such that $\mathrm{X}_{\mathrm{m}} \cap \mathrm{Z} \subset \mathrm{H}_{\mathrm{m}}$ and $\mu\left(\mathrm{H}_{\mathrm{m}}\right)<\eta_{\mathrm{m}}$. We notice that since $\cup_{\xi_{i} \in X_{m}}\left[u_{i}, v_{i}\right] \subset H_{m}$ we have $\mu\left(\cup_{\xi_{i} \in X_{m}}\left[u_{i}, v_{i}\right]\right)<\eta_{m}$ and

$$
\begin{aligned}
\sum_{i} D\left(\tilde{F}_{n}\left(\left[u_{i}, v_{i}\right]\right), \tilde{0}\right) & \leqslant \sum_{m=1}^{\infty} \sum_{\xi_{i} \in X_{m}} D\left(\tilde{F}_{n}\left(\left[u_{i}, v_{i}\right]\right), \tilde{0}\right) \\
& \leqslant \sum_{m=1}^{\infty} \frac{\varepsilon}{2^{m}}=\varepsilon
\end{aligned}
$$

and the statement is proved.

Lemma 3.16 ([15]). If $\tilde{\mathrm{f}}_{\mathfrak{n}}(\mathrm{x}) \rightarrow \tilde{\mathrm{f}}(\mathrm{x})$ a.e. in $[\mathrm{a}, \mathrm{b}]$ as $\mathrm{n} \rightarrow \infty$ where each $\tilde{\mathrm{f}}_{\mathfrak{n}}(\mathrm{x})$ is measurable on $[\mathrm{a}, \mathrm{b}]$, then for every $\eta>0$ there exists an open set $\mathrm{G}$ with $|\mathrm{G}|<\eta$ such that $\tilde{f}_{n}$ converges uniformly to $\tilde{f}$ on $[\mathrm{a}, \mathrm{b}] \backslash \mathrm{G}$.

By Egoroff's Theorem, we get the following lemma.

Lemma 3.17. Let $\tilde{f}_{n}:[a, b] \rightarrow \mathbb{R}_{\mathrm{F}}$ be a sequence of strong fuzzy Henstock integrable functions with primitives $\tilde{\mathrm{F}}_{\mathrm{n}}$. Suppose that $\lim _{\mathrm{n} \rightarrow \infty} \tilde{\mathrm{f}}_{\mathrm{n}}(\mathrm{x})=\tilde{\mathrm{f}}(\mathrm{x})$ for all $\mathrm{x} \in[\mathrm{a}, \mathrm{b}]$. Assume that there exists a sequence of measurable sets $X_{m} \subset[a, b]$ such that $\cup_{m} X_{m}=[a, b]$ and $\tilde{F}_{n}$ is uniformly strong $\operatorname{AC}^{\nabla}\left(X_{m}\right)$. Then there exists a sequence of closed sets $G_{m}, G_{m} \subset G_{m+1}$ such that $\mu\left([a, b] \backslash \cup_{m} G_{m}\right)=0$ and for any $m \in \mathbb{N}$ the sequence $\tilde{f}_{n}$ converges uniformly to $\tilde{\text { f on }} \mathrm{G}_{\mathrm{m}}$ and the sequence $\tilde{\mathrm{F}}_{\mathrm{n}}$ is uniformly strong $A C^{\nabla}\left(\mathrm{G}_{\mathrm{m}}\right)$ for $\mathrm{m} \in \mathbb{N}$.

Theorem 3.18. Let $\tilde{f}_{\mathrm{n}}:[\mathrm{a}, \mathrm{b}] \rightarrow \mathbb{R}_{\mathrm{F}}$ be a sequence of strong fuzzy Henstock integrable functions such that $\lim _{n \rightarrow \infty} \tilde{f}_{n}(x)=\tilde{f}(x)$ for all $x \in[a, b]$. Let $\tilde{F}_{n}$ be the primitives of $\tilde{f}_{n}$. Assume that there exists a sequence of measurable sets $X_{m} \subset[a, b]$ such that $\cup_{m} X_{m}=[a, b]$ and $\tilde{F}_{n}$ is uniformly strong $A C^{\nabla}\left(X_{m}\right)$. Then the sequence $\tilde{f}_{\mathrm{n}}$ is strong fuzzy Henstock equi-integrable on $[\mathrm{a}, \mathrm{b}]$.

Proof. By Lemma 3.17, there exists a sequence of closed sets $G_{m}, G_{m} \subset G_{m+1}$ such that $\mu\left([a, b] \backslash \cup_{m} G_{m}\right)=$ 0 and for any $m \in \mathbb{N}$ the sequence $\tilde{f}_{n}$ converges uniformly to $\tilde{f}$ on $G_{m}$ and the sequence $\tilde{F}_{n}$ is uniformly strong $A C^{\nabla}\left(G_{m}\right)$ for $m \in \mathbb{N}$. Denote $S=[a, b] \backslash \cup_{m} G_{m}$, then $\mu(S)=0$. Let $\varepsilon>0$ and put $\varepsilon_{m}=\frac{\varepsilon}{2^{m+4}(1+b-a)}$ for $m \in \mathbb{N}$. By the uniform convergence $\tilde{f}_{n} \rightarrow \tilde{f}$ on $G_{m}$, for every $m \in \mathbb{N}$ there exists $r_{m} \in \mathbb{N}$ such that

$$
\mathrm{D}\left(\tilde{\mathrm{f}}_{\mathrm{n}}(x), \tilde{f}_{l}(x)\right)<\varepsilon_{\mathrm{m}},
$$


where $n, l \geqslant r_{m}$ and $x \in G_{m}$. Since $\tilde{f}_{n}$ are strong fuzzy Henstock integrable, there exist $\delta_{m}^{1}(\xi)$ such that

$$
\sum_{i} \mathrm{D}\left(\tilde{\mathrm{F}}\left(\left[u_{i}, v_{i}\right]\right), \tilde{f}_{n}\left(\xi_{i}\right)\left(v_{i}-u_{i}\right)\right)<\varepsilon_{m},
$$

for any $\delta_{m}^{1}$-fine division $\left\{\left(\xi_{i},\left[u_{i}, v_{i}\right]\right)\right\}$. Since the sequence $\tilde{F}_{n}$ is uniformly strong $A C^{\nabla}\left(X_{m}\right)$, there exists $\delta_{m}^{2}(\xi)>0$ and $\eta_{m}>0$ such that

$$
\left|\sum_{i} \mathrm{D}\left(\tilde{\mathrm{F}}_{\mathfrak{n}}\left(\left[u_{i}, v_{i}\right]\right), \tilde{0}\right)-\sum_{j} \mathrm{D}\left(\tilde{\mathrm{F}}_{\mathfrak{n}}\left(\left[s_{j}, \mathrm{t}_{j}\right]\right), \tilde{0}\right)\right|<\varepsilon_{m},
$$

for $n \in \mathbb{N}$, if $\left\{\left(\xi_{i},\left[u_{i}, v_{i}\right]\right)\right\}$ and $\left\{\left(\zeta_{j},\left[s_{j}, t_{j}\right]\right)\right\}$ are arbitrary $\eta_{m}-$ close $\delta_{m}^{2}$-fine division with $\xi_{i}, \zeta_{j} \in G_{m}$.

Set $G_{0}=\emptyset$ and by the proof of Lemma 3.14 for each $m \in \mathbb{N}$ choose an open set $H_{m}$ such that $X_{m} \subset H_{m}, \mu\left(H_{m} \backslash X_{m}\right)<\frac{\eta_{m}}{2}$. There is a function $\delta^{*}(\xi)>0$ such that $B\left(\xi, \delta^{*}(\xi)\right) \subset H_{m} \backslash X_{m}$ for $\xi \in X_{m} \backslash X_{m-1}$ and $J \subset B\left(\zeta, \delta^{*}(\zeta)\right)$ if $\{(\zeta, J)\}$ is a $\delta^{*}$-fine division. By Lemma 3.12, there exists $\delta_{3}(\xi)>0$ such that

$$
\sum_{i} \mathrm{D}\left(\tilde{\mathrm{f}}_{\mathrm{n}}\left(\xi_{i}\right)\left(v_{\mathrm{i}}-\mathrm{u}_{\mathrm{i}}\right), \tilde{0}\right)<\frac{\varepsilon}{4}
$$

for any $\delta_{3}$-fine division with $\xi_{i} \in S$. By Lemma 3.14, there exists $\delta_{4}(\xi)>0$ and for any $\delta_{4}$-fine division such that

$$
\sum_{i} \mathrm{D}\left(\tilde{\mathrm{F}}_{\mathrm{n}}\left(\left[\mathrm{u}_{\mathrm{i}}, v_{\mathrm{i}}\right]\right), \tilde{0}\right)<\frac{\varepsilon}{4} .
$$

Let us now take $\delta(\xi) \leqslant \min \left\{\delta_{m}^{1}(\xi), \delta_{m}^{2}(\xi), \delta^{*}(\xi)\right\}$ for $\xi \in X_{m} \backslash X_{m-1}$ and $\delta(\xi) \leqslant \min \left\{\delta_{3}(\xi), \delta_{4}(\xi)\right\}$ for $\xi \in \mathrm{S}$. Next, we shall prove

$$
\sum_{i} D\left(\tilde{\mathrm{F}}_{n}\left(\left[u_{i}, v_{i}\right]\right), \tilde{f}_{n}\left(\xi_{i}\right)\left(v_{i}-u_{i}\right)\right)<\varepsilon
$$

In fact, by (3.4) and (3.5) we have

$$
\begin{aligned}
\sum_{i} \mathrm{D}\left(\tilde{\mathrm{F}}_{n}\left(\left[u_{i}, v_{i}\right]\right), \tilde{f}_{n}\left(\xi_{i}\right)\left(v_{i}-u_{i}\right)\right)= & \sum_{m} \sum_{\xi_{i} \in X_{m} \backslash X_{m-1}} \mathrm{D}\left(\tilde{F}_{n}\left(\left[u_{i}, v_{i}\right]\right), \tilde{f}_{n}\left(\xi_{i}\right)\left(v_{i}-u_{i}\right)\right) \\
& +\sum_{\xi_{i} \in S} \mathrm{D}\left(\tilde{F}_{n}\left(\left[u_{i}, v_{i}\right]\right), \tilde{f}_{n}\left(\xi_{i}\right)\left(v_{i}-u_{i}\right)\right) \\
\leqslant & \sum_{m} \sum_{\xi_{i} \in X_{m} \backslash X_{m-1}} \mathrm{D}\left(\tilde{F}_{n}\left(\left[u_{i}, v_{i}\right]\right), \tilde{f}_{n}\left(\xi_{i}\right)\left(v_{i}-u_{i}\right)\right) \\
& +\sum_{\xi_{i} \in S} \mathrm{D}\left(\tilde{F}_{n}\left(\left[u_{i}, v_{i}\right]\right), \tilde{0}\right)+\sum_{\xi_{i} \in S} \mathrm{D}\left(\tilde{f}_{n}\left(\xi_{i}\right)\left(v_{i}-u_{i}\right), \tilde{0}\right) \\
\leqslant & \sum_{m} \sum_{\xi_{i} \in X_{m} \backslash X_{m-1}} \mathrm{D}\left(\tilde{F}_{n}\left(\left[u_{i}, v_{i}\right]\right), \tilde{f}_{n}\left(\xi_{i}\right)\left(v_{i}-u_{i}\right)\right)+\frac{\varepsilon}{2} .
\end{aligned}
$$

Fix $m \in \mathbb{N}$. If $n \leqslant r_{m}$ then by (3.2), we have

$$
\sum_{\xi_{i} \in X_{m} \backslash X_{m-1}} D\left(\tilde{F}_{n}\left(\left[u_{i}, v_{i}\right]\right), \tilde{f}_{n}\left(\xi_{i}\right)\left(v_{i}-u_{i}\right)\right)<\varepsilon_{m}<\frac{\varepsilon}{2} .
$$

Suppose $n>r_{m}$, there exists a $\delta_{5}^{n}(\xi)>0$ and for any $\delta_{5}^{n}$-fine division such that

$$
\sum_{i} D\left(\tilde{F}_{n}\left(\left[u_{i}, v_{i}\right]\right), \tilde{f}_{n}\left(\xi_{i}\right)\left(v_{i}-u_{i}\right)\right)<\varepsilon_{m} .
$$

Let $\delta^{\prime}(\xi) \leqslant \min \left\{\delta(\xi), \delta_{5}^{n}(\xi)\right\}$ for $\xi \in[a, b]$. Then we have a $\delta^{\prime}$-fine division $\left\{\left(\zeta_{j},\left[s_{j}, t_{j}\right]\right)\right\}$ with $\zeta_{j} \in$ $X_{m} \backslash X_{m-1}$ such that $\left\{\left(\xi_{i},\left[u_{i}, v_{i}\right]\right)\right\}$ and $\left\{\left(\zeta_{j},\left[s_{j}, t_{j}\right]\right)\right\}$ are $\eta_{m}$-close. By (3.3) we have

$$
\left|\sum_{\xi_{j} \in X_{m} \backslash X_{m-1}} \mathrm{D}\left(\tilde{F}_{n}\left(\left[u_{i}, v_{i}\right]\right), \tilde{0}\right)-\sum_{j} \mathrm{D}\left(\tilde{F}_{n}\left(\left[s_{j}, t_{j}\right]\right), \tilde{0}\right)\right|<\varepsilon_{m},
$$


and by (3.8)

$$
\sum_{j} D\left(\tilde{F}_{n}\left(\left[s_{i}, t_{i}\right]\right), \tilde{f}_{n}\left(\zeta_{j}\right)\left(t_{i}-s_{i}\right)\right)<\varepsilon_{m}
$$

Now, using (3.7), (3.8), (3.9), (3.10) and (3.1) we have

$$
\begin{array}{rl}
\sum_{\xi_{i} \in X_{m} \backslash X_{m-1}} & D\left(\tilde{F}_{n}\left(\left[u_{i}, v_{i}\right]\right), \tilde{f}_{n}\left(\xi_{i}\right)\left(v_{i}-u_{i}\right)\right) \\
\leqslant & \left|\sum_{\xi_{i} \in X_{m} \backslash X_{m-1}} D\left(\tilde{F}_{n}\left(\left[u_{i}, v_{i}\right]\right), \tilde{0}\right)-\sum_{j} D\left(\tilde{F}_{n}\left(\left[s_{i}, t_{i}\right]\right), \tilde{0}\right)\right| \\
& +\sum_{j} D\left(\tilde{F}_{n}\left(\left[s_{i}, t_{i}\right]\right), \tilde{f}_{n}\left(\zeta_{j}\right)\left(t_{i}-s_{i}\right)\right)+\sum_{j} D\left(\tilde{f}_{n}\left(\zeta_{j}\right)\left(t_{i}-s_{i}\right), \tilde{f}_{r_{m}}\left(\zeta_{j}\right)\left(t_{i}-s_{i}\right)\right) \\
& +\sum_{j} D\left(\tilde{f}_{r_{m}}\left(\zeta_{j}\right)\left(t_{i}-s_{i}\right), \tilde{\mathrm{r}}_{r_{m}}\left(\left[s_{i}, t_{i}\right]\right)\right) \\
& +\left|\sum_{j} D\left(\tilde{F}_{r_{m}}\left(\left[s_{j}, t_{j}\right]\right), \tilde{0}\right)-\sum_{\xi_{i} \in X_{m} \backslash X_{m-1}} D\left(\tilde{F}_{r_{m}}\left(\left[u_{i}, v_{i}\right]\right), \tilde{0}\right)\right| \\
& +\sum_{\xi_{i} \in X_{m} \backslash X_{m-1}} D\left(\tilde{F}_{r_{m}}\left(\left[u_{i}, v_{i}\right]\right), \tilde{f}_{r_{m}}\left(\xi_{i}\right)\left(v_{i}-u_{i}\right)\right) \\
& +\sum_{\xi_{i} \in X_{m} \backslash X_{m-1}} D\left(\tilde{f}_{r_{m}}\left(\xi_{i}\right)\left(v_{i}-u_{i}\right), \tilde{f}_{n}\left(\xi_{i}\right)\left(v_{i}-u_{i}\right)\right) \\
< & 5 \varepsilon_{m}+2 \varepsilon_{m}(b-a)<\frac{7 \varepsilon}{2^{m+4}} .
\end{array}
$$

Hence by (3.6) we have

$$
\begin{aligned}
\sum_{i} \mathrm{D}\left(\tilde{\mathrm{F}}_{\mathfrak{n}}\left(\left[u_{i}, v_{i}\right]\right), \tilde{f}_{n}\left(\xi_{i}\right)\left(v_{i}-u_{i}\right)\right) & \leqslant \sum_{m} \sum_{\xi_{i} \in X_{m} \backslash X_{m-1}} \mathrm{D}\left(\tilde{F}_{n}\left(\left[u_{i}, v_{i}\right]\right), \tilde{f}_{n}\left(\xi_{i}\right)\left(v_{i}-u_{i}\right)\right)+\frac{\varepsilon}{2} \\
& <\sum_{m} \frac{7 \varepsilon}{2^{m+4}}=\frac{7 \varepsilon}{16} \sum_{m} \frac{1}{2^{m}}+\frac{\varepsilon}{2}=\varepsilon .
\end{aligned}
$$

That is to say that the theorem is proved.

Using above lemmas and Theorem 3.18, we obtain the main outcomes in this section.

Theorem 3.19 (Generalized Convergence Theorem). Let $\tilde{f}_{\mathrm{n}}:[\mathrm{a}, \mathrm{b}] \rightarrow \mathbb{R}_{\mathrm{F}}$ be a sequence of strong fuzzy Henstock integrable functions such that $\lim _{n \rightarrow \infty} \tilde{f}_{n}(x)=\tilde{f}(x)$ for all $x \in[a, b]$. Let $\tilde{F}_{n}$ be the primitives of $\tilde{f}_{n}$. Assume that there exists a sequence of measurable sets $X_{m} \subset[a, b]$ such that $\cup_{m} X_{m}=[a, b]$ and $\tilde{F}_{n}$ is uniformly strong $A C^{\nabla}\left(X_{m}\right)$. Then the function $\tilde{f}(x)$ is strong fuzzy Henstock integrable and

$$
\lim _{n \rightarrow \infty} \int_{a}^{b} \tilde{f}_{n}(x) d x=\int_{a}^{b} \tilde{f}(x) d x .
$$

\section{The existence of generalized solution for discontinuous fuzzy differential equations}

Given a fuzzy differential equation of the form

$$
\left\{\begin{array}{l}
x^{\prime}=\tilde{\mathfrak{f}}(\mathrm{t}, x), \\
x(\mathrm{a})=x_{0}, \quad x_{0} \in \mathbb{R}_{\mathrm{F}}
\end{array}\right.
$$

where $\tilde{f}:[a, b] \times \mathbb{R}_{\mathrm{F}} \rightarrow \mathbb{R}_{\mathrm{F}}$ is strong fuzzy Henstock integrable.

Let $C\left(I, \mathbb{R}_{F}\right)$ be a fuzzy number space and $I=[a, b]$. 
Definition 4.1. A fuzzy-number-valued function $x(t) \in C\left(I, \mathbb{R}_{F}\right)$ is called a generalized solution of problem (4.1) under strong fuzzy Henstock integrability setting, for simplicity written as G-solution, if $x(t)$ is strong $A C^{\nabla}$ and GH-differentiable a.e. on I.

In other words, a solution of problem (4.1) is called (i)-solution if it is (i)-GH-differentiable and a solution of problem (4.1) is called (ii)-solution if it is (i)-GH-differentiable.

Theorem 4.2. Assume that $\mathrm{x}(\mathrm{t}) \in \mathrm{C}\left(\mathrm{I}, \mathbb{R}_{\mathrm{F}}\right)$ is a (i)-solution (or (ii)-solution) of the initial value problems (4.1), if and only if $\tilde{\mathrm{f}}(\mathrm{t}, \mathrm{x}(\mathrm{t})) \in \mathrm{SFH}(\mathrm{I})$ and satisfied integral equation

$$
x(t)=x_{0}+\int_{t_{0}}^{t} f(s, x(s)) d s
$$

or

$$
x_{0}=x(t) \ominus_{\mathrm{H}}\left(-\int_{\mathrm{t}_{0}}^{\mathrm{t}} \mathrm{f}(\mathrm{s}, \mathrm{x}(\mathrm{s})) \mathrm{ds}\right),
$$

on some interval $\left(\mathrm{t}_{0}, \mathrm{t}\right) \subset \mathbb{R}$, under the strong differentiability condition, (i) or (ii), respectively.

Proof. $(\Rightarrow)$ Let $x(t) \in C\left(I, \mathbb{R}_{F}\right)$, then $x(t)$ is a generalized solution of problem (4.1), that is to say that $x(t)$ is strong $A C^{\nabla}$ and (i)-differentiable a.e. on I and satisfies $x^{\prime}(t)=\tilde{f}(t, x(t))$. By Theorem 3.11, $\tilde{f}(t, x(t))$ is strong fuzzy Henstock integrable on I. By the N-L formula of fuzzy integral (see [2]), we have

$$
x(t) \ominus_{\mathbf{H}} x\left(t_{0}\right)=\int_{t_{0}}^{t} x^{\prime}(t) d t .
$$

Thus, we have

$$
x(t)=x\left(t_{0}\right)+\int_{t_{0}}^{t} \tilde{f}(t) d t .
$$

$(\Leftarrow)$ By Theorem 3.11, $x(t)$ is (i)-GH-differentiable a.e. on I and satisfies $x^{\prime}(t)=\tilde{f}(t, x(t))$. That is, $x(t)$ is a generalized solution of problem (4.1).

For the same reasons, $x^{\prime}(t)$ is (ii)-GH-differentiable, the conclusion also holds.

Before proposing the existence theorems, we are going to propose the following theorem which helps us to prove the mentioned theorems.

Lemma 4.3 ([4]). If $\tilde{\mathrm{f}}, \tilde{\mathrm{g}}: \rightarrow \mathbb{R}_{\mathrm{F}}$ are strong differentiable on $(\mathrm{a}, \mathrm{b})$, then $\tilde{\mathrm{f}}+\tilde{\mathrm{g}}$ is strongly generalized differentiable on $(\mathrm{a}, \mathrm{b})$ and $(\tilde{\mathrm{f}}+\tilde{\mathrm{g}})^{\prime}(\mathrm{x})=\tilde{\mathrm{f}}^{\prime}(\mathrm{x})+\tilde{\mathrm{g}}^{\prime}(\mathrm{x})$.

Lemma 4.4 ([4]). If $\mathrm{g}:(\mathrm{a}, \mathrm{b}) \rightarrow \mathbb{R}$ is differentiable on $(\mathrm{a}, \mathrm{b})$ such that $\mathrm{g}^{\prime}$ has at most a finite number of roots in $(\mathrm{a}, \mathrm{b})$ and $\mathrm{c} \in \mathbb{R}_{\mathrm{F}}$, then $\tilde{\mathrm{f}}(\mathrm{x})=\mathrm{c} \cdot \mathrm{g}(\mathrm{x})$ is strongly generalized differentiable on $(\mathrm{a}, \mathrm{b})$ and $\tilde{f}^{\prime}(\mathrm{x})=\mathrm{c} \cdot \mathrm{g}^{\prime}(\mathrm{x})$.

Theorem 4.5. Let $\tilde{\mathrm{f}}:[\mathrm{a}, \mathrm{b}] \rightarrow \mathbb{R}_{\mathrm{F}}$.

(1) If $\tilde{f}(t)$ is strong $A C^{\nabla}$ on $[a, b]$, then $e^{t} \tilde{f}(t)$ is strong $A C^{\nabla}$ on $[a, b]$;

(2) if $\tilde{\mathrm{f}}(\mathrm{t})$ is strong $G H$-differentiable on $(\mathrm{a}, \mathrm{b})$,

then $e^{\mathrm{t}} \tilde{\mathrm{f}}(\mathrm{t})[\mathrm{a}, \mathrm{b}]$ is strong GH-differentiable on $(\mathrm{a}, \mathrm{b})$ and

$$
\left(e^{t} \tilde{f}(t)\right)^{\prime}=e^{t} \tilde{f}^{\prime}(t)+e^{t} \tilde{f}(t) .
$$

Proof. (1) If $\tilde{f}(t)$ is strong $A C^{\nabla}$ on $[a, b]$, for every $\varepsilon>0$, there exist $\varepsilon / e^{b}>0$ and $\eta>0$ such that for every finite sequence of non-overlapping intervals $\left\{\left[a_{i}, b_{i}\right]\right\}$ satisfying $\sum_{i}\left(b_{i}-a_{i}\right)<\eta$, we have

$$
\sum_{i} \sup _{a_{i} \leqslant t_{1} \leqslant t_{2} \leqslant b_{k}} D\left(\tilde{f}\left(t_{1}\right), \tilde{f}\left(t_{2}\right)\right)<\varepsilon / e^{b} .
$$


For above $\varepsilon>0$, because $e^{t}$ is $A C$ on $[a, b]$, there exists $\eta_{0}>0$, such that for every finite sequence of non-overlapping intervals $\left\{\left[a_{i}, b_{i}\right]\right\}_{i}$ satisfying $\sum_{i}\left(b_{i}-a_{i}\right)<\eta_{0}$, we have

$$
\sum_{i}\left|e^{b_{i}}-e^{a_{i}}\right|<\varepsilon / M
$$

where $M=\sup _{t \in[a, b]} D(\tilde{f}(t), \tilde{0})$. We put $\eta_{n}=\min \left\{\eta, \eta_{0}\right\}$ on each $S_{n}$ satisfying $\sum_{i}\left(b_{i}-a_{i}\right)<\eta_{n}$, we have

$$
\begin{aligned}
\sum_{k} \sup _{a_{i} \leqslant t_{1} \leqslant t_{2} \leqslant b_{k}} D\left(e^{t_{1}} \tilde{f}\left(t_{1}\right), e^{t_{2}} \tilde{f}\left(t_{2}\right)\right) & \leqslant \sum_{k} e^{b} \sup _{a_{i} \leqslant t_{1} \leqslant t_{2} \leqslant b_{k}} D\left(\tilde{f}\left(t_{1}\right), \tilde{f}\left(t_{2}\right)\right)+\sum_{k} D\left(e^{t_{1}} \tilde{f}\left(t_{1}\right), e^{t_{2}} \tilde{f}\left(t_{2}\right)\right) \\
& \leqslant e^{b} \sum_{k} \sup _{a_{i} \leqslant t_{1} \leqslant t_{2} \leqslant b_{k}} D\left(\tilde{f}\left(t_{1}\right), \tilde{f}\left(t_{2}\right)\right)+H(\tilde{f}(t), \tilde{0}) \sum_{k} \mid e^{b_{k}}-e^{a_{k} \mid} \\
& \leqslant \varepsilon+\varepsilon=2 \varepsilon .
\end{aligned}
$$

(2) From Lemma 4.3 and Lemma 4.4, we can prove it easily.

Theorem 4.6. If $\tilde{f}:[a, b] \rightarrow \mathbb{R}_{\mathrm{F}}$ is (SFH) integrable on $[a, b]$, then $e^{t} \tilde{f}(t)$ is $(S F H)$ integrable on $[a, b]$.

Proof. Since $\tilde{f}(t)$ is $(\mathrm{SFH})$ integrable on $[a, b]$, by Theorem 3.11, $\tilde{\mathrm{F}}(\mathrm{t})$ is strong $A C^{\nabla}$, such that $\tilde{\mathrm{F}}(\mathrm{t})$ is strong GH-differentiable on $[a, b]$ and $\tilde{F}^{\prime}(t)=\tilde{f}(t)$ a.e.. From Theorem 3.7, $e^{t} \tilde{F}(t)$ is strong $A C^{\nabla}$ and strong GH-differentiable on $[a, b]$. Applying Theorem 3.11 again, we get $e^{t} \tilde{f}(t)$ is $(S F H)$ integrable on $[a, b]$.

Theorem 4.7. Assume $x(\mathrm{t}) \in \mathrm{C}\left(\mathrm{I}, \mathbb{R}_{\mathrm{F}}\right)$ is a generalized (i)-solution (or (ii)-solution) of the initial value problem (4.1), if and only if there exists $M>0$ and satisfies integral equation

$$
x(t)=e^{-M\left(t-t_{0}\right)} \chi_{0}+\int_{t_{0}}^{t} e^{-M(t-s)}(\tilde{f}(s, x(s))+M x(s)) d s,
$$

or

$$
x(t)=e^{-M\left(t-t_{0}\right)} x_{0} \ominus\left(-\int_{t_{0}}^{t} e^{-M(t-s)}(\tilde{f}(s, x(s))+M x(s)) d s\right) .
$$

Proof. $(\Rightarrow)$ If $x(t)$ is a generalized (i)-solution of the initial value problem (4.1), then $x(t)$ is strong $A C^{\nabla}$ and (i)-GH-differentiable a.e. on I, satisfies $x^{\prime}(t)=\tilde{f}(t, x(t))$. By Theorem 4.5, $e^{M t} x(t)$ is strong $A C^{\nabla}$ and (i)-GH-differentiable a.e. on I, satisfies $\left(e^{M t} x(t)\right)^{\prime}=e^{M t}(\tilde{f}(t, x(t)))+M x(t)$. By Theorem 3.11, the function $e^{M t}(\tilde{f}(t, x(t)))+M x(t)$ is $(S F H)$ integrable on I. By Theorem 4.6, $\tilde{f}(t, x(t)) \in S F H(I)$ and satisfies integral equation

$$
x(t)=x_{0}+\int_{t_{0}}^{t} \tilde{f}(s, x(s)) d s .
$$

According to the second mean valued theorem of fuzzy number valued function, we have

$$
\int_{\mathfrak{t}_{0}}^{\mathrm{t}} e^{M s}(\tilde{f}(s, \chi(s))+(M x(s))) \mathrm{d} s=e^{M t} \chi(t) \ominus_{H} e^{M t_{0}} \chi_{0} .
$$

That is to say that

$$
x(t)=e^{-M\left(t-t_{0}\right)} \chi_{0}+\int_{t_{0}}^{t} e^{-M(t-s)}(\tilde{f}(s, x(s))+M x(s)) d s .
$$

$(\Leftarrow)$ If $x(t)$ satisfies integral equation in assumption, then $e^{M t} x(t)$ is strong $A C^{\nabla}$ and (i)-GH-differentiable a.e. on I, satisfies

$$
e^{M t} x(t)=e^{M t_{0}} \chi_{0}+\int_{t_{0}}^{t} e^{M s}(\tilde{f}(s, x(s))+M x(s)) d s
$$


By Theorem 4.7, $x(t)$ is strong $A C^{\nabla}$ and (i)-GH-differentiable a.e. on I, satisfies

$$
M e^{M t} \chi(t)+e^{M t} \chi^{\prime}(t)=e^{M t}(\tilde{f}(t, x(t))+M x(t)),
$$

that is $x^{\prime}(t)=\tilde{f}(t, x(t))$. So, $x(t)$ is a generalized (i)-solution of the initial value problem (4.1).

For the same reasons, $\chi^{\prime}(t)$ is (ii)-GH-differentiable, the conclusion also holds.

Theorem 4.8. If $\tilde{\mathrm{f}}:[\mathrm{a}, \mathrm{b}] \times \mathbb{R}_{\mathrm{F}} \rightarrow \mathbb{R}_{\mathrm{F}}$ satisfies conditions:

(1) there exist $u_{0}, v_{0} \in C\left(I, \mathbb{R}_{F}\right)$ and $u_{0} \preceq v_{0}$ such that $\tilde{f}\left(t, u_{0}(t)\right)$ and $\tilde{f}\left(t, v_{0}(t)\right)$ is strong fuzzy Henstock integrable on $\mathrm{I}$, where $\mathrm{u}_{0}, v_{0}$ is the upper and lower solution of problem (4.1), respectively and satisfies

$$
u^{\prime}(t) \preceq \tilde{f}\left(t, u_{0}(t)\right), u_{0}\left(t_{0}\right) \preceq x_{0}, \quad \text { and } \quad v^{\prime}(t) \succeq \tilde{f}\left(t, v_{0}(t)\right), v_{0}\left(t_{0}\right) \succeq x_{0} ;
$$

(2) there exist $M, L>0$, for every $x_{1}, x_{2} \in\left[u_{0}, v_{0}\right]$,

$$
-M\left(x_{2} \ominus_{H} x_{1}\right) \preceq \tilde{f}\left(t, x_{2}\right) \ominus_{H} \tilde{f}\left(t, x_{1}\right) \preceq L\left(x_{2} \ominus_{H} x_{1}\right),
$$

for $x_{1} \preceq x_{2}$;

(3) for $\tilde{\mathrm{f}}(\mathrm{t}, \mathrm{x}): \mathrm{I} \times \mathbb{R}_{\mathrm{F}} \rightarrow \mathbb{R}_{\mathrm{F}}$ such that $\tilde{\mathrm{f}}(\mathrm{x})=\tilde{\mathrm{f}}(\mathrm{t}, \mathrm{x})$ mapping $\mathrm{C}\left(\mathrm{I}, \mathbb{R}_{\mathrm{F}}\right)$ to $\mathrm{SFH}(\mathrm{I})$, and $\mathrm{u}_{0}^{\prime}(\mathrm{t}), v_{0}^{\prime}(\mathrm{t}) \in \mathrm{SFH}(\mathrm{I})$.

Then there exist a generalized (i)-solution (or (ii)-solution) of the problem (4.1) on $\left[\mathfrak{u}_{0}, v_{0}\right]$.

Proof. Define the operator as following:

$$
(A x)(t)=e^{-M\left(t-t_{0}\right)} x_{0}+\int_{t_{0}}^{t} e^{-M(t-s)}(\tilde{f}(s, x(s))+M x(s)) d s
$$

or

$$
(A x)(t)=e^{-M\left(t-t_{0}\right)} \chi_{0} \ominus_{H}\left(-\int_{t_{0}}^{t} e^{-M(t-s)}(\tilde{f}(s, x(s))+M x(s)) d s\right),
$$

where $x(t)$ is $(\mathfrak{i})$-GH-differentiable or $(\mathfrak{i})$-GH-differentiable. We only prove case of $(\mathfrak{i})$-GH-differentiable here.

By Theorem 4.7, $x(t)$ is a generalized (i)-solution of the initial value problem (4.1), it is equivalent to $x$ is a fixed point of $A$. By condition (2) and definition of $A$, we get that $A$ is a increasing operator.

Since $u_{0}(t)$ is a lower solution of problem (4.1) and strong GH-differentiable, by Theorem 4.5, we have

$$
\begin{aligned}
\left(\mathfrak{u}_{0}(t) e^{M t}\right)^{\prime} & =e^{M t} u_{0}^{\prime}(t)+M e^{M t} u_{0}(t) \\
& \preceq e^{M t}\left(\tilde{f}\left(t, u_{0}(t)\right)+M u_{0}(t)\right) .
\end{aligned}
$$

Now taking the integral both sides, we have

$$
e^{M t} u_{0}(t) \ominus_{H} e^{M t_{0}} u_{0}\left(t_{0}\right) \preceq \int_{t_{0}}^{t} e^{M s}\left(\tilde{f}\left(s, u_{0}(s)\right)+M u_{0}(s)\right) d s .
$$

So,

$$
\begin{aligned}
\mathrm{u}_{0}(\mathrm{t}) \preceq & e^{-M\left(t-t_{0}\right)} x_{0}+\int_{t_{0}}^{t} e^{-M(t-s)}\left(\tilde{f}\left(s, u_{0}(s)\right)\right. \\
& \left.+M u_{0}(s)\right) d s=\left(A u_{0}\right)(t) .
\end{aligned}
$$

From the same reason, we have $\left(A v_{0}\right)(t) \preceq v_{0}(t)$. Next, we put an iterative sequence:

$$
u_{n}=A u_{n-1}, \quad v_{n}=A v_{n-1}, \quad n=1,2, \cdots,
$$


then we have

$$
\begin{aligned}
\mathfrak{u}_{0} \preceq u_{1} \preceq u_{2} \preceq \cdots & \preceq u_{n} \preceq \cdots \preceq v_{n} \\
& \preceq \cdots \preceq v_{2} \preceq v_{1} \preceq v_{0} .
\end{aligned}
$$

For all $t \in I$, by condition (2), we have

$$
\begin{aligned}
v_{n}(t) \ominus_{H} u_{n}(t)=\left(A v_{n-1}\right)(t) \ominus_{H}\left(A u_{n-1}\right)(t)= & \int_{t_{0}}^{t} e^{-M(t-s)}\left[\left(\tilde{f}\left(s, v_{n-1}(s)\right) \ominus_{H} \tilde{f}\left(s, u_{n-1}(s)\right)\right)\right. \\
& \left.+M\left(v_{n-1}(s) \ominus_{H} u_{n-1}(s)\right)\right] d s \\
\leqslant & \int_{t_{0}}^{t} e^{-M(t-s)}(M+L)\left(v_{n-1}(s) \ominus_{H} u_{n-1}(s)\right) d s \\
\leqslant & (M+L) D\left(\int_{t_{0}}^{t} v_{n-1}(s) d s, \int_{t_{0}}^{t} u_{n-1}(s) d s\right) .
\end{aligned}
$$

By condition (3) and the controlled convergence theorem for real-valued Henstock integral, there exists an $\mathrm{N} \in \mathbb{N}$, we have

$$
\begin{aligned}
H\left(v_{n}(t), u_{n}(t)\right) & \leqslant N(M+L) D\left(\int_{t_{0}}^{t} v_{n-1}(s) d s, \int_{t_{0}}^{t} u_{n-1}(s) d s\right) \\
& \leqslant N(M+L)(H) \int_{t_{0}}^{t} H\left(v_{n-1}(s), u_{n-1}(s)\right) d s .
\end{aligned}
$$

That is

$$
\mathrm{H}\left(v_{\mathrm{n}}(\mathrm{t}), \mathrm{u}_{\mathrm{n}}(\mathrm{t})\right) \leqslant \frac{\left[\mathrm{N}(\mathrm{M}+\mathrm{L})\left(\mathrm{t}-\mathrm{t}_{0}\right)\right]^{\mathrm{n}}}{\mathrm{n} !} \mathrm{H}\left(v_{0}, \mathrm{u}_{0}\right) .
$$

For this expression we have $\mathrm{H}\left(v_{n}(t), u_{n}(t)\right) \rightarrow 0$ for $n \rightarrow \infty$.

We get that $u_{m} \ominus_{H} u_{n} \preceq v_{m} \ominus_{H} u_{n} \preceq v_{n} \ominus_{H} u_{n}$, when $m>n$, that is, $H\left(u_{m}, u_{n}\right) \leqslant N \cdot H\left(v_{n}, u_{n}\right)$. So, $\left\{u_{n}\right\}$ is a Cauchy sequence. We have $u_{n} \rightarrow x$. From the same reasons, $v_{n} \rightarrow y$. Thus, we have $H(x, y)=0$. This implies $x(t)=y(t)$. That is to say that $x_{0}(t)=x(t)=y(t)$ is a fixed point of $A$, By Theorem 4.2, $x_{0}(t)$ is a generalized (i)-solution of the initial value problem (4.1).

Theorem 4.9. Let a fuzzy-number-valued function $\tilde{\mathrm{f}}:[\mathrm{a}, \mathrm{b}] \times \mathbb{R}_{\mathrm{F}} \rightarrow \mathbb{R}_{\mathrm{F}}$ fulfils condition

(1) $\tilde{f}(\cdot, t)$ is continuous for $t \in[a, b]$;

(2) $\int_{a}^{b} \tilde{f}(s, x(s)) d$ s exists for every $x \in \mathbb{R}_{F}$;

(3) Let $\{x(t): t \in[a, b]\}$ be the family of continuous functions,

$$
\left\{F_{x}(t)=\int_{a}^{b} \tilde{f}(s, x(s)) d s: x \in\{x(t)\}\right\},
$$

is uniformly strong $A C^{\nabla}$ for each $\mathrm{x}$ and $\left\{\mathrm{F}_{\mathrm{x}}(\mathrm{t})\right\}$ is equi-continuous on $[\mathrm{a}, \mathrm{b}]$.

Then problem (4.1) has a generalized (i)-solution (or (ii)-solution).

Proof. We only prove case of $(\mathfrak{i})$-GH-differentiable. Since $\left\{\mathrm{F}_{x}(\mathrm{t})\right\}$ is equicontinuous on $[\mathrm{a}, \mathrm{b}]$ and $x \in$ $\{x(t)\}, F_{x}(a)=\tilde{0}$, we have $\left\{F_{x}(t)\right\}$ is uniformly bounded on $[a,+\infty)$. Put $\left|\left\{F_{x}(t)\right\}\right| \leqslant M$ and $B \subset C\left(I, \mathbb{R}_{F}\right)$. We define:

$$
\mathrm{H}(\varphi(\mathrm{t}), \psi(\mathrm{t}))=\sup _{\mathrm{t} \in[\mathrm{a},+\infty)} \mathrm{D}(\varphi(\mathrm{t}), \psi(\mathrm{t})),
$$

for every $\varphi(t), \psi(t) \in B$ and $x_{0} \in \mathbb{R}_{\mathrm{F}}$. 
Consider $\mathrm{K}=\left\{\mathrm{x}, \mathrm{x} \in \mathrm{B}, \mathrm{D}\left(\mathrm{x}, \mathrm{x}_{0}\right) \leqslant \mathrm{M}\right\}$ and integral operator as following:

$$
(T x)(t)=x_{0}+\int_{a}^{b} \tilde{f}(s, x(s)) d s .
$$

Then $\mathrm{K}$ is a bounded closed convex set on B. In fact, we have

$$
\begin{aligned}
H\left(T x, x_{0}\right) & =\sup _{t \in[a,] b} D\left(\int_{a}^{b} \tilde{f}(s, x(s)) d s, \tilde{0}\right) \\
& =\sup _{t \in[a,+b]} D\left(F_{x}(t), \tilde{0}\right) \leqslant M .
\end{aligned}
$$

So, $\mathrm{T}(\mathrm{K}) \subset \mathrm{K}$.

Next, we shall prove $T$ is continuous. Let $x_{n}(t), x(t) \in K, n=1,2, \cdots$, and $x_{n}(t) \longrightarrow x(t)$. By continuity of $\tilde{f}$, we have

$$
\lim _{n \rightarrow \infty} \tilde{f}\left(t, x_{n}(t)\right)=\tilde{f}(t, x(t)), \quad t \in[a, b] .
$$

By condition (3), $\left\{F_{n}(t)=\int_{a}^{b} \tilde{f}\left(s, x_{n}(s)\right) d s, x_{n} \in\{x(t)\}\right\}$ is uniformly strong $A C^{\nabla}$ for $x$ and $\left\{F_{n}(t)\right\}$ is equi-continuous on $[a, b]$, so, it is uniformly bounded. By Ascoli-Arzela's theorem, $\left\{F_{n}(t)\right\}$ is uniformly convergence on $[a, b]$. By Theorem 3.19, we have

$$
\lim _{n \rightarrow \infty} \int_{a}^{b} \tilde{f}\left(s, x_{n}(s)\right) d s=\int_{a}^{b} \tilde{f}(s, x(s)) d s .
$$

That is,

$$
\begin{aligned}
H\left(T x_{n}, T x\right)= & \sup _{t \in[a, b]} D\left(\int_{a}^{b} \tilde{f}\left(s, x_{n}(s)\right) d s, \int_{a}^{b} \tilde{f}(s, x(s)) d s\right) \\
& +\sup _{t \in[a, b]} D\left(F_{n}(t), F_{x}(t)\right) \longrightarrow 0 .
\end{aligned}
$$

This implication implies $T$ is continuous.

At last, we will prove $T$ is a completely continuous operator. In fact, $T(N)=\left\{F_{x}(t), x \in N\right\}$ is a family of continuous function on $[a, b]$ for every bounded subset $N \subset K$. From condition (3), $T(N)$ is equi-continuous and uniformly bounded. Since $T(N)$ is a relative compact set on $K$, we obtain that $T$ is a completely continuous operator.

By Schauder's fixed point theorem, $T$ has a fixed point $x(t) \in K$ such that $T x(\cdot)=x(\cdot)$, i.e., $x(t)=$ $x_{0}+\int_{a}^{b} \tilde{f}(s, x(s)) d s$ which is a generalized (i)-solution of problem (4.1).

To illustrate, we consider the following example:

Example 4.10. Let

$$
\tilde{\mathrm{F}}(\mathrm{t})= \begin{cases}\tilde{\mathrm{A}} \cdot \mathrm{t}^{2} \sin \frac{1}{\mathrm{t}^{2},} & \mathrm{t} \neq 0 \\ 0, & \mathrm{t}=0\end{cases}
$$

and

$$
\tilde{A}(s)= \begin{cases}s, & 0 \leqslant s \leqslant 1 \\ 2-s, & 1<s \leqslant 2, \\ 0, & \text { others. }\end{cases}
$$

Then $\tilde{F}^{\prime}(t)$ is SFH integrable but not Kaleva integrable. Consider the fuzzy differential equation as following:

$$
\chi^{\prime}=\tilde{f}(t, \chi(t))=\tilde{g}(t, \chi(t))+\tilde{F}^{\prime}(t),
$$


where $\tilde{g}(t, x(t))=\tilde{A} \cdot t^{2} x$. Then Cauchy problem

$$
\left\{\begin{array}{l}
x^{\prime}=\tilde{A} \cdot\left(t^{2} x+2 t \sin \frac{1}{t^{2}}-\frac{2}{t} \cos \frac{1}{t^{2}}\right) \\
x(a)=x_{0}, \quad x_{0} \in \mathbb{R}_{F}
\end{array}\right.
$$

has a generalized (i)-solution

$$
x(t)=x_{0}+\tilde{A} \cdot \int_{0}^{t}\left(t^{2} x+2 t \sin \frac{1}{t^{2}}-\frac{2}{t} \cos \frac{1}{t^{2}}\right) d t .
$$

\section{Conclusions}

The major contribution of this paper is to study the problems of existence of generalized solutions to discontinuous fuzzy systems such as with right-hand function is strong fuzzy Henstock integral under generalized differentiability. Some known results of fuzzy differential equations are extended, which might be helpful in the analysis of dynamic systems with uncertainties.

\section{Acknowledgment}

The authors thank to the National Nature Science Foundation of China (Grant No. 11671001, No. 61472056 and No. 11461062), the PhD Research Startup Foundation of Chongqing University of Posts and Telecommunications (No. A2014-90, A2016-13) and Chongqing Municipal Science and technology project (cstc2015jcyjA00015).

\section{References}

[1] R. Alikhani, F. Bahrami, Global solutions of fuzzy integro-differential equations under generalized differentiability by the method of upper and lower solutions, Inform. Sci., 295 (2015), 600-608. 1

[2] B. Bede, S. G. Gal, Generalizations of the differentiability of fuzzy-number-valued functions with applications to fuzzy differential equations, Fuzzy Sets and Systems, 151 (2005), 581-599. 2, 2.1, 4

[3] B. Bede, S. G. Gal, Solutions of fuzzy differential equations based on generalized differentiability, Commun. Math. Anal., 9 (2010), 22-41. 1

[4] B. Bede, I. J. Rudas, A. L. Bencsik, First order linear fuzzy differential equations under generalized differentiability, Inform. Sci., 177 (2007), 1648-1662. 1, 4.3, 4.4

[5] Y. Chalco-Cano, H. Román-Flores, On new solutions of fuzzy differential equations, Chaos Solitons Fractals, 38 (2008), 112-119. 2

[6] S. S. L. Chang, L. A. Zadeh, On fuzzy mapping and control, IEEE Trans. Systems, Man, and Cybernet., SMC-2 (1972), 30-34. 1

[7] M.-H. Chen, C.-S. Han, Some topological properties of solutions to fuzzy differential systems, Inform. Sci., 197 (2012), 207-214. 1

[8] M.-H. Chen, C.-S. Han, Periodic behavior of semi-linear uncertain dynamical systems, Fuzzy Sets and Systems, 230 (2013), 82-91. 1

[9] P. Diamond, P. Kloeden, Metric spaces of fuzzy sets, Theory and applications, World Scientific Publishing Co., Inc., River Edge, NJ, (1994). 2

[10] D. Dubois, H. Prade, Towards fuzzy differential calculus, I, Integration of fuzzy mappings, Fuzzy Sets and Systems, 8 (1982), 1-17. 1, 2

[11] D. Dubois, H. Prade, Towards fuzzy differential calculus, II, Integration on fuzzy intervals, Fuzzy Sets and Systems, 8 (1982), 105-116. 1

[12] D. Dubois, H. Prade, Towards fuzzy differential calculus, III, Differentiation, Fuzzy Sets and Systems, 8 (1982), 225-233. 1

[13] Z.-T. Gong, On the problem of characterizing derivatives for the fuzzy-valued functions, II, Almost everywhere differentiability and strong Henstock integral, Fuzzy Sets and Systems, 145 (2004), 381-393. 1, 3.3

[14] Z.-T. Gong, Y.-B. Shao, Global existence and uniqueness of solutions for fuzzy differential equations under dissipative-type conditions, Comput. Math. Appl., 56 (2008), 2716-2723. 1

[15] Z.-T. Gong, Y.-B. Shao, The controlled convergence theorems for the strong Henstock integrals of fuzzy-number-valued functions, Fuzzy Sets and Systems, 160 (2009), 1528-1546. 1, 3.2, 3.3, 3.5, 3.6, 3, 3.16

[16] R. Henstock, Theory of integration, Butterworths, London, (1963). 1 
[17] O. Kaleva, Fuzzy differential equations, Fuzzy Sets and Systems, 24 (1987), 301-319. 1, 2

[18] O. Kaleva, The Cauchy problem for fuzzy differential equations, Fuzzy Sets and Systems, 35 (1990), 389-396. 1

[19] P. E. Kloeden, T. Lorenz, Fuzzy differential equations without fuzzy convexity, Fuzzy Sets and Systems, 230 (2013), 65-81. 1

[20] J. Kurzweil, Generalized ordinary differential equations and continuous dependence on a parameter, (Russian) Czechoslovak Math. J., 7 (1957), 418-449. 1

[21] J. Kurzweil, J. Jarník, Equi-integrability and controlled convergence of Perron-type integrable functions, Real Anal. Exchange, 17 (1991/92), 110-139. 3.8, 3.12

[22] V. Lakshmikantham, R. N. Mohapatra, Theory of fuzzy differential equations and inclusions, Series in Mathematical Analysis and Applications, Taylor \& Francis, Ltd., London, (2003). 1

[23] P. Y. Lee, Lanzhou lectures on Henstock integration, Series in Real Analysis, World Scientific Publishing Co., Inc., Teaneck, NJ, (1989). 1, 3.1

[24] M. Mosleh, M. Otadi, Approximate solution of fuzzy differential equations under generalized differentiability, Appl. Math. Model., 39 (2015), 3003-3015. 1

[25] M. L. Puri, D. A. Ralescu, Differentials of fuzzy functions, J. Math. Anal. Appl., 91 (1983), 552-558. 1

[26] M. L. Puri, D. A. Ralescu, Fuzzy random variables, J. Math. Anal. Appl., 114 (1986), 409-422. 2

[27] S. Seikkala, On the fuzzy initial value problem, Fuzzy Sets and Systems, 24 (1987), 319-330. 1

[28] M. Sugeno, Theory of fuzzy integrals and its applications, PhD thesis, Tokyo Institute of Technology, Tokyo, (1974). 1

[29] E. J. Villamizar-Roa, V. Angulo-Castillo, Y. Chalco-Cano, Existence of solutions to fuzzy differential equations with generalized Hukuhara derivative via contractive-like mapping principles, Fuzzy Sets and Systems, 265 (2015), 24-38. 1

[30] Z. Y. Wang, G. J. Klir, Fuzzy measure theory, Plenum Press, New York, (1992). 1

[31] C.-X. Wu, Z.-T. Gong, On Henstock integrals of interval-valued functions and fuzzy-valued functions, Fuzzy Sets and Systems, 115 (2000), 377-391. 1

[32] C.-X. Wu, Z.-T. Gong, On Henstock integral of fuzzy-number-valued functions, I, Fuzzy Sets and Systems, 120 (2001), 523-532. 1

[33] X.-P. Xue, Y.-Q. Fu, Carathéodory solutions of fuzzy differential equations, Fuzzy Sets and Systems, 125 (2002), $239-243$. 1

[34] L. Zadeh, Fuzzy sets, Information and Control, 3 (1965), 338-353. 1 